large rubella outbreak with spread from the workplace to the community. JAMA 2000;284:2733-2739.

\section{Gene Therapy Reverses Sepsis-Induced Suppression of Lung Antibacterial Host Defense}

Sepsis syndrome frequently is complicated by the development of nosocomial infections, particularly gram-negative pneumonia. Although TNF-alpha (TNF) has been shown to mediate many of the pathophysiologic events in sepsis, this cytokine is a critical component of innate immune response within the lung. Chen and coinvestigators from University of Michigan Medical School, Ann Arbor, and Department of Microbiology, Toho University, Tokyo, Japan, hypothesized that the transient transgenic expression of TNF within the lung during the postseptic period could augment host immunity against nosocomial pathogens. To test this, mice underwent 26-gauge cecal ligation and puncture (CLP) as a model of abdominal sepsis, followed 24 hours later by intratracheal administration of Pseudomonas aeruginosa. In animals undergoing sham surgery followed by bacterial challenge, $P$ aeruginosa were almost completely cleared from the lungs within 24 hours. In contrast, mice undergoing CLP were unable to clear $P$ aeruginos $a$ and rapidly developed bacteremia.

Alveolar macrophages (AM) recovered from mice 24 hours after CLP produced significantly less TNF ex vivo, as compared with AM from sham animals. Furthermore, the adenoviral mediated transgenic expression of TNF within the lung increased survival in CLP animals challenged with $P$ aeruginosa: from $25 \%$ in animals receiving control vector to $91 \%$ in animals administered recombinant murine TNF adenoviral vector. Improved survival in recombinant murine TNF adenoviral vector-treated mice was associated with enhanced lung bacterial clearance and proinflammatory cytokine expression, as well as enhanced AM phagocytic activity and cytokine expression when cultured ex vivo. These observations suggest that intrapulmonary immunostimulation with TNF can reverse sepsis-induced impairment in antibacterial host defense.

FROM: Chen GH, Reddy RC, Newstead MW, Tateda K, Kyasapura BL, Standiford TJ. Intrapulmonary TNF gene therapy reverses sepsis-induced suppression of lung antibacterial host defense. J Immunol 2000;165:6496-6503.

\section{New Recombinant BCG Vaccines Provide Greater TB Immunity}

Horwitz and coworkers, from the School of Medicine, University of California, Los Angeles, California, have developed new recombinant bacillus Calmette-Guérin (BCG) vaccines that offer greater immunity than current commercially available vaccines. Using two commercially available strains of BCG as host strains, BCG Connaught and Tice, the investigators constructed two recombinant $\mathrm{BCG}$ vaccines stably expressing and secreting the $30 \mathrm{kDa}$ major secretory protein of Mycobacterium tuberculosis. They tested the efficacy of the two strains in the highly susceptible guinea pig model of pulmonary TB, a model noteworthy for its close resemblance to human TB.

Animals immunized with the recombinant $B C G$ vaccines and challenged by aerosol with a highly virulent strain of $M$ tuberculosis had 0.5 logs fewer $M$ tuberculosis in their lungs and $1 \log$ fewer bacilli in their spleens on average than animals immunized with their parental conventional BCG vaccine counterparts. Statistically, these differences were highly significant. Paralleling these results, at necropsy, animals immunized with the recombinant BCG vaccines had fewer and smaller lesions in the lung, spleen, and liver and significantly less lung pathology than animals immunized with the parental $B C G$ vaccines.

The recombinant vaccines are the first vaccines against TB more potent than the current commercially available BCG vaccines, which were developed nearly a century ago.

FROM: Horwitz MA, Harth G, Dillon BJ, MaslesaGalic. Recombinant bacillus Calmette-Guérin ( $\mathrm{BCG}$ ) vaccines expressing the Mycobacterium tuberculosis $30-\mathrm{kDa}$ major secretory protein induce greater protective immunity against tuberculosis than conventional BCG vaccines in a highly susceptible animal model. Proc Natl Acad Sci USA 2000;97:13853-13858.

\section{Increase in TB Among Foreign-Born Persons in US, 1993-1998}

Immigration is a major force sustaining the incidence of TB in the United States. Data from case reports on TB were used to describe trends and characteristics of foreign-born persons with TB and the implications for TB program planning and policy development.

The number of TB cases among foreign-born persons increased $2.6 \%$, from 7,402 in 1993 to 7,591 in 1998; the proportion of US cases that were foreign-born increased from $29.8 \%$ to $41.6 \%$. During 1993 to 1998 , the TB case rate was 32.9 per 100,000 population in foreign-born persons, compared with 5.8 per 100,000 in US-born persons. Six states reported $73.4 \%$ of foreign-born cases (California, New York, Texas, Florida, New Jersey, and Illinois). Approximately two thirds of these cases were originally from Mexico, the Philippines, Vietnam, India, China, Haiti, and South Korea. Among those for whom date of US entry was known, $51.5 \%$ arrived 5 years or less prior to the diagnosis of TB. Most were male and aged 25 to 44 years. During 1993 to 1996 , the proportion receiving some portion of treatment under directly observed therapy increased from $27.3 \%$ to $59.1 \%$; approximately $70 \%$ completed therapy in 12 months. The rate of primary resistance to isoniazid was $11.6 \%$ and to both isoniazid and rifampin was $1.7 \%$.

As the United States moves toward the goal of TB elimination, success will depend increasingly on reducing the impact of TB in foreign-born persons. Continued efforts to tailor local TB control strategies to the foreign-born community and commitment to the global TB battle are essential.

FROM: Talbot EA, Moore M, McCray E, Binkin NJ. Tuberculosis among foreign-born persons in the United States, 1993-1998. JAMA 2000;284:2894-2900. 